\title{
Dynamics of the Quantum Discord with Weak Measurement for a Two-atom System in Thermal Reservoirs
}

\author{
Mei Bai, Hong Jia Xu, Xue Qun Yan* \\ School of Physical Science and Technology, Tian Gong University, Tianjin, China \\ Email address: \\ yanxuequn@tiangong.edu.cn (Xue Qun Yan) \\ ${ }^{*}$ Corresponding author
}

To cite this article:

Mei Bai, Hong Jia Xu, Xue Qun Yan. Dynamics of the Quantum Discord with Weak Measurement for a Two-atom System in Thermal Reservoirs. American Journal of Modern Physics. Vol. 9, No. 5, 2020, pp. 68-72. doi: 10.11648/j.ajmp.20200905.11

Received: September 23, 2020; Accepted: November 5, 2020; Published: November 11, 2020

\begin{abstract}
Weak measurement is a kind of state partial collapse measurement developed on the basis of von Neumann measurement and positive operator value measurement, which allows us to explore the quantum world which has the least influence on the research system. Based on the weak measurement theory, the dynamics of quantum discord for two isolated atoms in their own thermal reservoirs is presented. We examine the time evolution of both standard quantum discord and quantum discord with weak measurement for the two-atom system, and analyzes the differences between the standard quantum discord and quantum discord with weak measurement in the evolution process with time, as well as the general role of the strength parameter in determing the discord and affecting its dynamic evolution. We show that quantum discords depend on how weak or strong one perturbs the quantum system. We also show that the difference of the standard quantum discord and the quantum discord with weak measurements increases as the strength parameter decreases. This means that the weak measurements can capture more quantum discord of a bipartite system. Our results show that the weak measurement performed on one of the subsystems can lead to the quantum discord that is a more natural measure of quantum correlations than the standard quantum discord captured by the projective measurements.
\end{abstract}

Keywords: Weak Measurement, Quantum Discord with Weak Measurement, Two-atom System

\section{Introduction}

The development of quantum information and quantum computing over the last two decades has led to increasing attention to quantum correlations [1-8]. In [1], the author introduces a new computational model DQC1, and proves that DQC1 only needs one qubit of information, which can bring the possibility of the presence of quantum discord that quantifier quantum correlations in a bipartite system. In [2-4], discussed and studied the related nonclassical aspects of separable states revealed by quantum discord, and revealed the conversion between quantum and classical. Specifically, in [5-7], the quantum discord in the two-atom system is analyzed, and the relationship between classical correlation and quantum correlation is compared and analyzed from the perspective of quantum discord. This is because quantum correlations are basic resources to allow one to perform tasks that would be more difficult [9-11]. Each of the types of correlations can be useful for specific tasks. Among them, entanglement is the most famous kind of quantum correlation. However, it has been shown that quantum discord is more general and important than entanglement for the fundamentals of physics and for quantum information processing tasks. For instance, quantum discord is a fundamental resource for deterministic one-qubit quantum computation and remote state preparation [12-14]. In addition, a relation of quantum discord with quantum communication has also been pointed out, such as in local broadcasting [15] and quantum state merging [16, 17]. More interestingly, some studies have shown that the dynamics of quantum discord can maintain a constant value in the long-time limit even when entanglement suddenly disappears [18].

The original context in which Ollivier and Zurek introduced quantum discord is by discussing the disturbance caused on one subsystem of a bipartite state due to projective measurements performed on the other [19]. According to traditional interpretation of quantum mechanics, measurement of the quantum system collapses it into a new state that is different from the one presented before the measurement. Thus, the 
measurement in quantum mechanics is usually a procedure that destroys many properties of the system. However, weak measurements were introduced as a theoretical scheme [20], allowing us to probe into the quantum system with minimum effects caused to the system of study [21-26]. Quantum correlations with weak measurement is an emerging research subject. Their properties are different from those by the means of projective measurement [27]. Another interesting subject is to investigate the dynamics of different kinds of correlations in noisy environments. The knowledge of the dynamic behavior of correlations will help us design suitable protocols to protect correlations during information processing. The inevitable interaction between a quantum system and its environment would destroy quantum correlations between the system and lead to the reduction of a useful resource. Strategies for preventing the decay of quantum correlations, and thus to fight decoherence are important for effective realization of quantum information processing. Therefore, understanding the dynamics of quantum correlations between subsystems in noisy environments is essential for quantum information and quantum computation [28].

Despite the fact that the correlation dynamics has been broadly studied for the quantum systems in dissipation environments, the problem is still open for effect of the environment on quantum discord with weak measurement. In this paper, we just focus on the two-atom system composed of two noninteractive two-level atoms, each trapped in a thermal reservoir. We will explore the dynamics of the quantum discord between the two atoms in the thermal environments. Our main goal is to investigate the influence of the strength parameter of measurement on the quantum discord by means of our next model. Our results show that the weak measurements can reveal more quantum correlations of bipartite quantum states under dissipation environments.

\section{Weak Measurement and Quantum Discord}

The standard quantum discord is captured by the projective measurements. The measurement of a multipartite quantum system with using projective measurement will result in coherence loss and quantum discord will be destroyed. However, weak quantum measurements, which was originally proposed by Aharonov, Albert and Vaidman (AAV) [20], may not lose the system coherence completely. The AAV results have been discussed in many papers and also experiments have been realized and confirmed their predictions [29, 30].

Before introducing the quantum discord with weak measurements, we briefly review the definition of the quantum discord with a local projective measurement. Given a bipartite quantum state $\rho_{A B}$ with $\rho_{A}\left(\rho_{B}\right)$ the reduced state of system $A(B)$, the standard quantum discord is defined as the difference between the total correlation and the classical correlation by the following expression

$$
D\left(\rho_{A B}\right)=I\left(\rho_{A B}\right)-Q\left(\rho_{A B}\right)
$$

Here, the total correlation between two subsystems $A$ and $B$ of the bipartite quantum system $\rho_{A B}$ is measured by quantum mutual information

$$
I\left(\rho_{A B}\right)=S\left(\rho_{A}\right)+S\left(\rho_{B}\right)-S\left(\rho_{A B}\right)
$$

and $S\left(\rho_{j}\right)$ is the von Neumann entropy entropy of the quantum state $\rho_{j}$, which is given by

$$
S\left(\rho_{j}\right)=-\operatorname{Tr}_{j}\left(\rho_{j} \log _{2} \rho_{j}\right)=-\sum_{i} \lambda_{j}^{i} \log _{2} \lambda_{j}^{i}
$$

Where the subscript $j$ indicates either the subsystem $A(B)$ or the total system, and $\left\{\lambda_{j}^{i}\right\}$ are the nonzero eigenvalues of the quantum state $\rho_{j} . Q\left(\rho_{A B}\right)$ is the classical correlation between the two subsystems [19]. As discussed in Ref. [31], the classical correlation is given by

$$
Q\left(\rho_{A B}\right)=S\left(\rho_{A}\right)-\min _{\left\{\Pi_{k}\right\}}\left[S\left(\rho_{A B} \mid\left\{\Pi_{k}\right\}\right)\right]
$$

where the minimum is taken over the complete set of orthonormal projectors $\left\{\prod_{k}=\left|\phi_{j}\right\rangle\left\langle\phi_{j}\right|,(j=1,2)\right\}$, where $\left|\phi_{1}\right\rangle=\cos \theta|0\rangle+e^{i \varphi} \sin \theta|1\rangle$ and $\left|\phi_{2}\right\rangle=e^{-i \varphi} \sin \theta|0\rangle-\cos \theta|1\rangle$, corresponding to measure one of the subsystems. $|0\rangle$ and $|1\rangle$ are known as computational basis states. $S\left(\rho_{A B} \mid\left\{\Pi_{k}\right\}\right)=\sum_{k} p_{k} S\left(\rho_{k}\right)$ is the quantum conditional entropy, $\rho_{k}=\operatorname{Tr}_{B}\left(\prod_{k} \rho_{A B} \Pi_{k}\right) / p_{k} \quad$ is the conditional density operator, and $p_{k}=\operatorname{Tr}_{A B}\left(\rho_{A B} \prod_{k}\right)$ is the probability associated with the measurement outcome $k$. Thus the quantum discord is defined

$$
D\left(\rho_{A B}\right)=S\left(\rho_{B}\right)-S\left(\rho_{A B}\right)+\min _{\left\{\Pi_{k}\right\}} S\left(\rho_{A B} \mid\left\{\Pi_{k}\right\}\right)
$$

We will calculate the quantum discord via numerical minimization over two independent real parameters $\theta$ and $\varphi$.

Indeed, we may extend this definition directly to the quantum discord with weak measurement by using the notion of weak measurement operators. The theory of weak measurements can be formulated in terms of measurement operator formalism as done by Oreshkov and Brun [22]. The weak measurement operators are given as

$$
P( \pm x)=\sqrt{\frac{1 \mp \tanh x}{2}} \Pi_{0}+\sqrt{\frac{1 \pm \tanh x}{2}} \Pi_{1}
$$

where $x$ is the strength parameter of measurement, $\Pi_{0}$ and $\Pi_{1}$ are orthogonal projectors that satisfy $\Pi_{0}+\Pi_{1}=I$. It is easy found that in the strong measurement limit we have the projective measurement operators, i.e., $\lim _{x \rightarrow \infty} P(+x)=\Pi_{1}$ and 
$\lim _{x \rightarrow \infty} P(-x)=\Pi_{0}$. The quantum discord with the weak measurement performed on the subsystem $B$ is defined as

$$
D_{w}\left(\rho_{A B}\right)=S\left(\rho_{B}\right)-S\left(\rho_{A B}\right)+\min _{\left\{\Pi_{k}\right\}} S_{w}\left(A \mid P^{B}(x)\right)
$$

where the weak quantum conditional entropy is given by

$$
S_{w}\left(A \mid P^{B}(x)\right)=P(+x) S\left(\rho_{A \mid P^{B}(x)}\right)+P(-x) S\left(\rho_{A \mid P^{B}(-x)}\right)
$$

with

$$
P( \pm x)=\operatorname{Tr}_{A B}\left[\left(I_{A} \otimes P^{B}( \pm x)\right) \rho_{A B}\left(I_{A} \otimes P^{B}( \pm x)\right)\right]
$$

and the post-measurement state for the subsystem $A$ is given by

$$
\rho_{A \mid P^{B}( \pm x)}=\frac{\operatorname{Tr}_{B}\left[\left(I_{A} \otimes P^{B}( \pm x)\right) \rho_{A B}\left(I_{A} \otimes P^{B}( \pm x)\right)\right]}{\operatorname{Tr}_{A B}\left[\left(I_{A} \otimes P^{B}( \pm x)\right) \rho_{A B}\left(I_{A} \otimes P^{B}( \pm x)\right)\right]}
$$

where $I_{A}$ is the identity operator on the Hilbert space $H_{A}$, and $P^{B}( \pm x)$ is the measurement operator performed on subsystem $B$.

\section{Description of the Model}

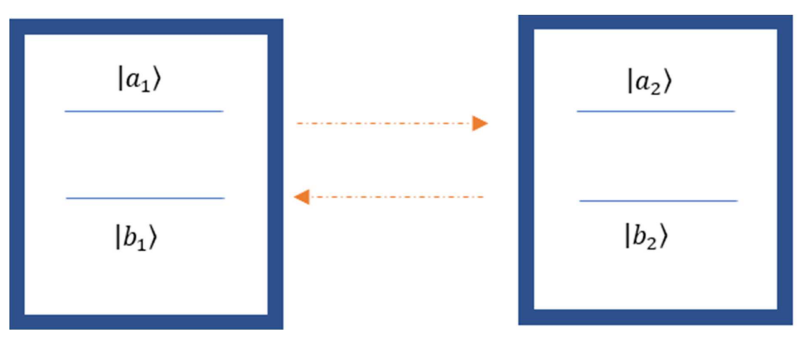

Figure 1. Two noninteractive two-level atoms, which are placed in two spatially separated thermal reservoirs and interact independently with thermal reservoirs with the same characteristics, have no direct interaction between atoms.

Two-atom system are primary building blocks for encoding correlations. Now we consider two noninteractive two-level atoms, which are independently interacting with identical thermal reservoirs. There is no direct interaction between the atoms. The physical model is shown in Figure 1. According to the general quantum reservoir theory [32], we can derive the following equation of motion for the reduced density matrix of the two-level atoms interacting with their local thermal reservoirs of mean thermal photon numbers $m$ and $n$.

$$
\begin{aligned}
\frac{d \rho}{d t}= & -\frac{1}{2} \gamma_{1}(m+1)\left[\sigma_{1}^{+} \sigma_{1} \rho-2 \sigma_{1} \rho \sigma_{1}^{+}+\rho \sigma_{1}^{+} \sigma_{1}\right] \\
& -\frac{1}{2} \gamma_{1} m\left[\sigma_{1} \sigma_{1}^{+} \rho-2 \sigma_{1}^{+} \rho \sigma_{1}+\rho \sigma_{1} \sigma_{1}^{+}\right] \\
- & \frac{1}{2} \gamma_{2}(n+1)\left[\sigma_{2}^{+} \sigma_{2} \rho-2 \sigma_{2} \rho \sigma_{2}^{+}+\rho \sigma_{2}^{+} \sigma_{2}\right]
\end{aligned}
$$

$$
-\frac{1}{2} \gamma_{2} n\left[\sigma_{2} \sigma_{2}^{+} \rho-2 \sigma_{2}^{+} \rho \sigma_{2}+\rho \sigma_{2} \sigma_{2}^{+}\right]
$$

Here $\gamma_{i}$ is the spontaneous emission rate of two-level atom $i(i=1,2)$, and $\sigma_{i}^{+}, \sigma_{i}$ are the usual Pauli raising and lowering operators of two-level atom $i$. Here we assume that the correlation time between the two-level atoms and the reservoirs is much shorter than the characteristic time of the dynamic evolution of the two-level atoms so that the Markov approximation is valid. In this case, memory effects in the reduced system dynamics can be ignored.

The solution of Eq.(11) depends on the initial state of the two-atom system. In the representation spanned by two-atom system produced states $|00\rangle,|01\rangle,|10\rangle,|11\rangle$, consider the following two-qubit initial state

$$
\rho_{A B}(0)=(1-P)|11\rangle\left\langle 11|+P| \varphi^{+}\right\rangle\left\langle\varphi^{+}\right|
$$

where $\left|\varphi^{+}\right\rangle=\frac{1}{\sqrt{2}}(|10\rangle+|01\rangle)$, and $0 \leq P \leq 1$. For this state, the Bell state $\left|\varphi^{+}\right\rangle$is mixed with the excited state $|11\rangle$. For $P=0$ it is separable state, and $P=1$ it is the maximal entanglement state $\rho_{A B}(0)=\left|\varphi^{+}\right\rangle\left\langle\varphi^{+}\right|$. For convenience of calculation, we take the simplest case of two-atom system, we assume $m=n$ and $\gamma_{1}=\gamma_{2}=\gamma$. The matrix elements of the solution of master Eq. (11) is given by

$$
\begin{aligned}
\rho_{11}(t)= & \frac{1}{2(2 n+1)^{2}}\left\{2 n^{2}-2 n[P(2 n+1)-2(n+1)] e^{-(2 n+1) \gamma t}\right. \\
& \left.-\left[P(n+1)(4 n+2)-2(n+1)^{2}\right] e^{-2(2 n+1) \gamma t}\right\}
\end{aligned}
$$

$$
\begin{gathered}
\rho_{22}(t)=\rho_{33}(t)=\frac{1}{2(2 n+1)^{2}}\{2 n(n+1)-[P(2 n+1)-2(n+1)] \\
\left.\times e^{-(2 n+1) \gamma t}+\left[P(n+1)(4 n+2)-2(n+1)^{2}\right] e^{-2(2 n+1) \gamma t}\right\} \\
\rho_{44}(t)=\frac{1}{2(2 n+1)^{2}}\left\{2(n+1)^{2}+2(n+1)[P(2 n+1)-2(n+1)]\right. \\
\left.\times e^{-(2 n+1) \gamma t}-\left[P(n+1)(4 n+2)-2(n+1)^{2}\right] e^{-2(2 n+1) \gamma t}\right\} \\
\rho_{23}(t)=\rho_{32}(t)=\frac{P}{2} e^{-(2 n+1) \gamma t}
\end{gathered}
$$

where the remaining matrix elements are zero.

\section{Dynamics of Quantum Correlations of the Two-atom System}

To examine the dynamical behavior of the quantum discord under weak measurement in dissipation environments, we will 
investigate the influence of the strength parameter of measurement and thermal effect on the quantum correlations by means of the dissipative model. In what follows, for several typical cases the dynamics of the standard quantum discord and the quantum discord with weak measurements are plotted as function of the scaled time $\gamma t$.

In figure 2 , the time evolution of the standard quantum discord and the quantum discord with weak measurements is shown for different values of the mean thermal photon number $n$ as $P$ and $x$ are fixed. First, we note that both of the quantum correlations disappear in the asymptotic limit in thermal reservoirs of nonzero mean photon number. This is a direct consequence of quantum system interacting with their dissipation environments. On the other hand, in order to see how the dissipation affects these two quantum correlations differently, we change greatly the mean photon number in the thermal reservoirs. In figures $2(a),(b)$ and (c), we compare the time evolution of the standard quantum discord and the quantum discord with weak measurements. As shown, under the given conditions for $P$ and $x$, the difference of both quantum correlations becomes larger when dissipation effect is weaker.

In the following we return to consider the effect of weak measurements on the time evolution of the correlations between two atoms. Let $P$ and $n$ be fixed. In figure 3, we give a description of how the time evolution of both the standard quantum discord and the quantum discord with weak

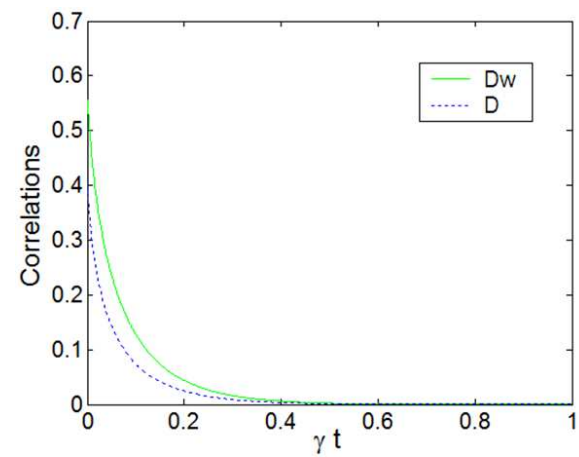

(a) $\mathrm{n}=2$

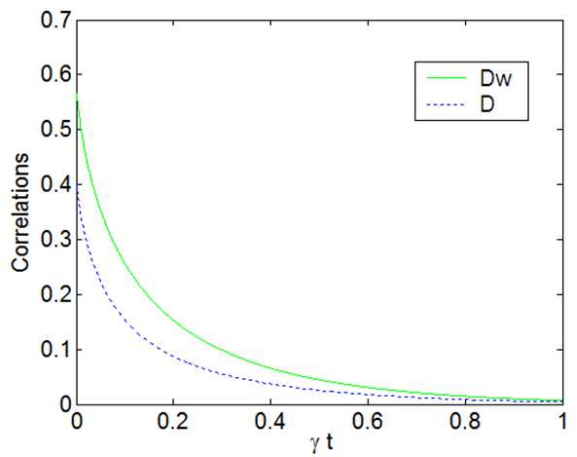

(b) $\mathrm{n}=0.5$ measurements for various values of the strength parameter $x$. From figure 3 we observe that the difference of the standard quantum discord and the quantum discord with weak measurements becomes small as $x$ increase. It can be expected that as $x \rightarrow \infty$, the quantum discord with weak measurements becomes the standard quantum discord under the Von Neumann measurement. Nevertheless, this extreme condition is not relevant to our purpose, since we are interested in the effect of weak measurements on quantum discord.

As a general behavior, all plots of figures 2 and 3 show that both of the quantum correlations tend to disappear in the asymptotic limit in thermal reservoirs. It is obvious that in the hot reservoir quantum correlations are easily destroyed. Therefore, one may deduce that the quantum correlations can be erased by increasing the thermal field parameter. Furthermore, we observe from the plots of figures 2 and 3 that the quantum discord with weak measurements is larger than the standard quantum discord for all time. This means that the weak measurement can capture more quantum correlations of a bipartite system than the strong (projective) measurement. In other words, if we perform quantum measurement weakly, then there can be more quantum correlations between the subsystems which can be exploited for practical use. In addition, these results also show that measurements do modify the dynamic of the quantum discord.

Figure 2. (Color online) Time evolution of the standard quantum discord (blue dotted line) and the quantum discord with weak measurement (green solid line) of the two-atom system as a function of dimensionless scaled time $\gamma t$ with $P=0.5$ and $x=0.5$. (a) $n=2 ;$ (b) $n=0.5 ;$ (c) $n=0.01$.

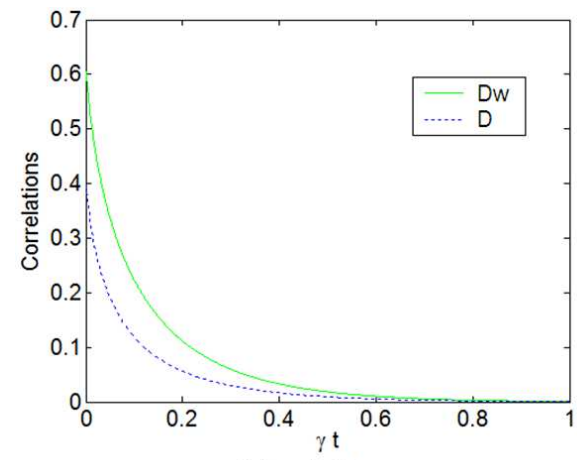

(a) $\mathrm{x}=0.01$

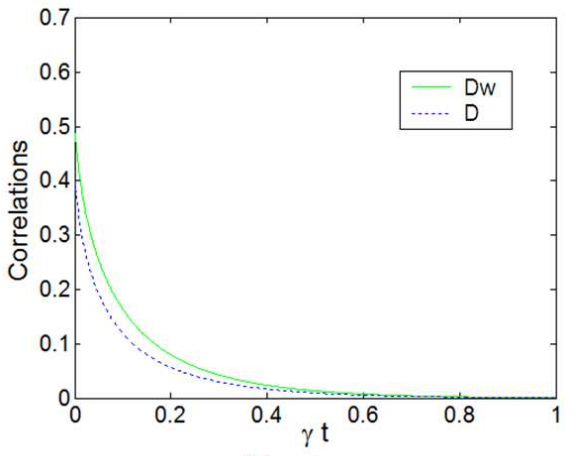

(b) $\mathrm{x}=1$

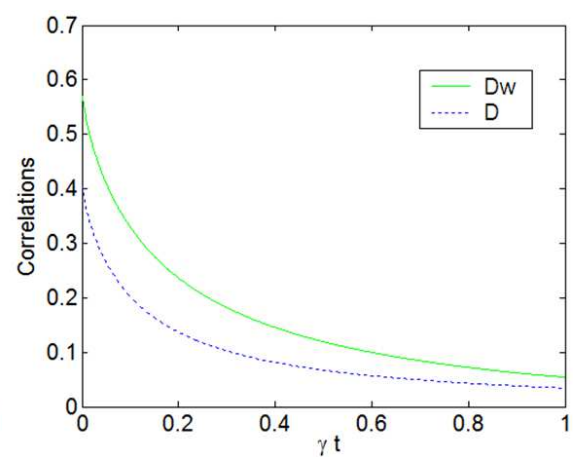

(c) $\mathrm{n}=0.01$

Figure 3. (Color online) Time evolution of the standard quantum discord (blue dotted line) and the quantum discord with weak measurement (green solid line) of the two-atom system as a function of dimensionless scaled time $\gamma t$ with $P=0.5$ and $n=1$. (a) $x=0.01 ;$ (b) $x=1 ;(c) \quad x=2$. 


\section{Conclusion}

Here we will summarize our results. We have studied the time evolution of the standard quantum discord and quantum discord with weak measurements for two isolated atoms, each in its own thermal reservoir. It is found that the difference between the standard quantum discord and the quantum discord with weak measurements tend to increase as the dissipation effects become weak, and both of the quantum correlations disappear in the asymptotic limit. Special attention has been paid for effect of weak measurements in the evolution of the quantum discord of two qubits in this work. It is shown that the difference of the standard quantum discord and the quantum discord with weak measurements increases as the strength parameters $x$ decreases. This is taken to imply that the dynamics of the quantum discord is sensitive to the measurements, and the weak measurements can capture more quantum discord of a bipartite system.

\section{References}

[1] E. Knill, R. Laflamme, "Power of One Bit of Quantum Information”, Phys. Rev. Lett, 81, 5672-5675, December 1998.

[2] L. C. Céleri, J. Maziero, J, R. M. Serra, "Theoretical and experimental aspects of quantum discord and related measures", Int. J. Quantum Inf, 09, 1837, July 2011.

[3] K. Modi, A. Brodutch, H. Cable, T. Paterek, V. Vedral, "The classical-quantum boundary for correlations: Discord and related measures”, Rev. Mod. Phys, 84, 1655-1707, November 2012.

[4] W. H. Zurek, "Quantum discord and Maxwell's demons", Phys. Rev. A, 67, 012320, January 2003.

[5] X. Q. Yan, Z. L. Yue, "Dynamics of quantum and classical correlations of a two-atom system in thermal reservoirs", Chaos, Solitons \& Fractals., 57, 117-122, December 2013.

[6] M. Ali, A. R. P. Rau, G. Alber, "Quantum discord for two-qubit X states”, Phys. Rev. A, 81, 042105, April 2010.

[7] S. Luo, "Quantum discord for two-qubit systems", Phys. Rev. A, 77, 042303, April 2008.

[8] G. Manzano, F. Plastina, R. Zambrini, "Optimal Work Extraction and Thermodynamics of Quantum Measurements and Correlations", Phys. Rev. Lett., 121, 120602, September 2018.

[9] E. Chitambar, G. Gour, "Quantum resource theories", Rev. Mod. Phys, 91, 025001, April 2019.

[10] A. Streltsov, G. Adesso, M. B. Plenio, "Colloquium: Quantum coherence as a resource”, Rev. Mod. Phys, 89, 041003 (34), October 2017.

[11] J. Ma, B. Yadin, D. Girolami, V. Vedral, M. Gu, "Converting Coherence to Quantum Correlations", Phys. Rev. Lett., 116, 160407, April 2016.

[12] E. Knill, R. Laflamme, "Power of One Bit of Quantum Information”, Phys. Rev. Lett., 81, 5672, December 1998.

[13] A. Datta, A. Shaji, C. M. Caves, "Quantum Discord and the Power of One Qub”, Phys. Rev. Lett., 100, 050502, February 2008.
[14] B. P. Lanyon, M. Barbieri, M. P. Almeida, A. G. White, "Experimental Quantum Computing without Entanglement", Phys. Rev. Lett., 101, 200501, November 2008.

[15] M. Piani, P. Horodecki, R. Horodecki, "No-Local-Broadcasting Theorem for Multipartite Quantum Correlations", Phys. Rev. Lett., 100, 090502, March 2008.

[16] D. Cavalcanti, L. Aolita, S. Boixo, K. Modi, M. Piani, A. Winter, "Operational interpretations of quantum discord", Phys. Rev. A, 83, 032324, March 2011.

[17] V. Mdhok, A. Datta, "Interpreting quantum discord through quantum state merging”, Phys. Rev. A, 83, 032323, March 2011.

[18] J. Wang, et al, Opt. Comm. 285, 2961, 2012.

[19] H. Olliver, W. H. Zurek, "Quantum Discord: A Measure of the Quantumness of Correlations", Phys. Rev. Lett., 88, 017901, December 2001.

[20] Y. Aharonov, D. Z. Albert, L. Vaidman, "How the result of a measurement of a component of the spin of a spin-1/2 particle can turn out to be 100", Phys. Rev. Lett., 60, 1351, April 1988.

[21] I. M. Duck, P. M. Stevenson, E. C. G. Sudarshan, "The sense in which a "weak measurement" of a spin- $\frac{1}{2}$ particle's spin component yields a value 100", Phys. Rev. D, 40, 2112, September 1989.

[22] O. Oreshkov, T. A. Brun, "Weak measurements are universal", Phys. Rev. Lett., 95, 110409, November 2005.

[23] M. Szyniszewski, A. Romito, H. Schomerus, "Entanglement transition from variable-strength weak measurements", Phys. Rev. B, 100, 064204, August 2019.

[24] Y. H. Chen, T. A. Brun, "Qubit positive-operator-valued measurements by destructive weak measurements", Phys. Rev. A, 99, 062121, June 2019.

[25] M. H. Wang, Q. Y. Cai, "High-fidelity quantum cloning of two nonorthogonal quantum states via weak measurements", Phys. Rev. A, 99, 012324, January 2019.

[26] L. Rosales-Zárate, B. Opanchuk, M. D. Reid, "Weak measurements and quantum weak values for NOON states", Phys. Rev. A, 97, 032123, March 2019.

[27] P. Wang, C. Chen, X. Peng, J. Wrachtrup, R. B. Liu, "Characterization of Arbitrary-Order Correlations in Quantum Baths by Weak Measurement", Phys. Rev. Lett., 123, 050603, August 2019.

[28] M. A. Nielsen, I. L. Chuang, "Quantum computation and Quantum Information”, Cambridge University press: Cambridge, 2000.

[29] N. W. M. Ritchie, J. G. Story, R. G. Hulet, "Realization of a measurement of a "weak value", Phys. Rev. Lett., 66, 1107-1110, March 1991.

[30] G. J. Pryde, J. L. O'Brien, A. G. White, T. C. Ralph, H. M. Wiseman, "Measurement of Quantum Weak Values of Photon Polarization", Phys. Rev. Lett., 94, 220405, June 2005.

[31] J. Maziero, L. C. Céleri, R. M. Serra, V. Vedral, "Classical and quantum correlations under decoherence", Phys. Rev. A, 80, 044102 , October 2009.

[32] M. O. Scully, M. S. Zubairy, Quantum Optics; Cambridge University Press: Cambridge, 1997. 